\title{
LAS RAZONES DE LA COHERENCIA
}

Claudio Michelon

La coherencia como tema de estudio forma hoy parte de las preocupaciones de los teóricos del derecho, del conocimiento, de la ética y la política, y de la metafísica, entre otros. Sin embargo, son raras las tentativas de una fertilización cruzada entre los avances hechos en cada uno de estos campos y el resultado de ese aislamiento es un gran número de oportunidades perdidas.

Esta es una de las grandes contribuciones de Amalia Amaya para el debate sobre la coherencia, una contribución que empezó antes del artículo que se publica en este volumen de Discusiones ${ }^{1}$. La otra gran

${ }^{1}$ Amaya, A. (2008) ‘Justification, Coherence, and Epistemic Responsibility in Legal Fact-finding' Episteme: A Journal of Social Epistemology Vol. 5 (3). 
contribución de Amaya reside en su intento de esclarecer complejas conexiones entre diversos aspectos del análisis de la coherencia. El resultado es un esbozo de una teoría general de la coherencia.

Mi comentario está centrado en dos problemas relacionados, a saber, los problemas del valor y de la insuficiencia de la coherencia como estándar de justificación racional. Sobre el valor de la coherencia, me parece que los argumentos generales presentados por Amaya, en particular el argumento epistémico, no son suficientes para justificar su conclusión. Aún más, existen argumentos que específicamente, en particular en el ámbito de la razón práctica, logran justificar con éxito el valor de la coherencia como criterio de racionalidad. Desarrollo este tema en la sección II más abajo.

Sobre el problema de la insuficiencia de la coherencia como criterio de racionalidad, tesis que es defendida en diversos pasajes del artículo de Amaya, mi preocupación está centrada en discutir qué otro criterio de racionalidad puede ser conjugado con la coherencia para producir resultados más racionales. Este tema se conecta directamente con una cuestión sobre la cual Amaya ha cambiado su opinión, a saber, la cuestión sobre el tipo de responsabilidad epistemológica del sujeto racional. Creo que hay razones para sostener que su posición original (ecléctica) puede ser una alternativa mejor que la postura adoptada en el artículo, a favor de las virtudes epistemológicas como herramienta que previene la manipulación del estándar racional de coherencia. La discusión sobre este tema ocupa la sección III más abajo.

Pero antes de tratar estos dos temas, es necesario tejer algunas consideraciones iniciales sobre las dos características centrales imbricadas en la idea de coherencia, a saber, no-contradicción y unidad. Esta breve discusión preliminar, que informará la discusión más interesante sobre el valor y la suficiencia de la coherencia, es el objeto de la próxima sección.

\section{I - Ventajas RACIONAles de la COHERENCiA}

El primer paso en mi argumento es la identificación de las potenciales ventajas de los argumentos coherentistas en procesos de 
justificación racional y, en particular, en la justificación jurídica. Hay fundamentalmente dos ventajas en la adopción de la coherencia como criterio de racionalidad: la coherencia es garantía de la consistencia (ya que la última es condición necesaria para que se obtenga la primera) y la existencia de coherencia entre diversas unidades aparentemente discretas afirma que éstas pueden ser tomadas como elementos de un conjunto formado intensionalmente, en otras palabras, es una garantía de unidad entre estos elementos. Cada una de estas dos tesis sobre las ventajas de la coherencia es controvertida en la literatura y, por esta razón, se hace necesario discutirlas, aunque brevemente, antes de entrar en lo substancial de mi argumento.

No hay duda de que hay una diferencia entre la coherencia y la simple consistencia entre proposiciones ${ }^{2}$. La consistencia es una cuestión de no contradicción entre proposiciones. No-contradicción es una condición del sentido de cualquier argumento, vale decir, cualquier argumento que contenga o implique proposiciones contradictorias, es necesariamente inválido ${ }^{3}$. Por lo tanto, la no contradicción es fundamentalmente todo o nada 4 . Pero la diferencia entre consistencia y coherencia no implica que una no esté contenida en la otra. De hecho, creo que ningún grupo de proposiciones puede ser considerado coherente si hay contradicciones entre los elementos cuya coherencia se discute.

La necesidad de eliminar contradicciones para que se pueda hablar de coherencia es una de las principales funciones de los mecanismos de contracción y de reinterpretación sugeridas en la tercera sección del trabajo, aquí discutido, de Amaya. La contracción permite

${ }^{2}$ MacCormick, N. (1978) Legal Reasoning and Legal Theory (Oxford, OUP), 152228.

${ }^{3}$ Como ya sostuviera Aristóteles Metafísica IV, 6 (1011b13-16). El surgimiento de las llamadas lógicas paraconsistentes no niega esta conclusión Aristotélica (o, por lo menos, no la niega necesariamente). La negación del principio lógico de la explosión puede ser considerado simplemente una herramienta que permite razonar sobre (y comparar) complejos sistemas que contienen una inconsistencia.

${ }^{4}$ En sentido contrario Wintgens, L. (2000) Droit, Principes et Théories: Pour un Positivisme Critique (Bruxelles, Bruylant), 102. 
evitar contradicciones por medio de la eliminación de una o más de las proposiciones contradictorias. La reinterpretación permite que se delimite el significado de las proposiciones en conflicto de modo tal de eliminar la contradicción. Por ejemplo, en el razonamiento jurídico una de las formas tradicionales de evitar contradicciones por reinterpretación es considerar que una de las proposiciones en conflicto se aplica generalmente mientras que la otra se aplica de forma más específica a ciertos casos como una excepción a la regla general.

La principal objeción a la tesis de que la coherencia supone la consistencia se funda en la creencia conforme a la cual la primera es una cuestión de grado que no puede ser sometida a la camisa de fuerza del todo o nada que implica la noción de consistencia. A pesar de esta objeción, no creo que haya un real conflicto entre la tesis de que la coherencia es gradual y la tesis de que la no-contradicción es una condición necesaria de la coherencia. La consistencia es solamente una condición necesaria, pero no una condición suficiente para la coherencia de las proposiciones. La otra condición necesaria para la coherencia, i.e. la unidad de los elementos que son coherentes, puede admitir distintos grados de satisfacción. Vale decir, una vez que la barrera "todo o nada" de la consistencia es vencida, hay espacio lógico para hablar de grados de coherencia.

La segunda condición necesaria para la coherencia de distintos elementos está conectada con la segunda ventaja racional de la coherencia. La coherencia requiere unidad de elementos. No basta que los elementos no se contradigan, es también necesario que ellos formen un conjunto. La formación de un conjunto, en este sentido, no se puede obtener con la simple identificación extensional de los elementos que forman parte del conjunto. Es también necesaria una conexión intensional entre los elementos. Esto significa que los elementos deben pertenecer al conjunto por una razón.

La búsqueda de esta conexión intensional es una indagación acerca del significado del conjunto que forman los elementos. Ese significado no puede ser atribuido externamente a cualquiera de estos elementos, pero debe ser un atributo intrínseco de los mismos. Los teóricos del derecho han hablado de la coherencia como resultado del hecho de que esta conexión intensional se encuentra en el hecho de que 
los elementos vinculan instrumental o intrínsecamente a un conjunto de valores comunes ${ }^{5}$. De hecho aquí se puede hablar de grados de coherencia, tanto del punto de vista puramente cuantitativo (¿cuántos elementos son explicables en términos de los valores comunes?) como del punto de vista cualitativo, cuando dos o más concepciones diferentes de los valores que dan unidad intensional a los elementos pueden ser consideradas mejores o peores explicaciones del significado de la vida humana o de uno de sus aspectos.

Dos cuestiones centrales para el debate sobre la coherencia (y estrechamente relacionadas) surgen de esta caracterización de las ventajas racionales de la coherencia en la razón práctica. En primer lugar, está la cuestión del valor de la coherencia para la razón práctica, vale decir, la pregunta sobre porqué no-contradicción y unidad deben ser consideradas ventajas del punto de vista de la razón práctica. En segundo lugar, surge la pregunta acerca del lugar de la coherencia en una teoría comprehensiva de la razón práctica. O sea, cuál es precisamente la contribución de los ideales de no-contradicción y de unidad de significado para una concepción adecuada de la razón práctica. Esos son dos de los problemas centrales discutidos por Amaya en su artículo. Del primero se ocupa la discusión de su séptima tesis, mientras que el segundo es, yo creo, la preocupación central de las tesis 3 y 4. La discusión de esos dos problemas es la preocupación principal del presente comentario.

\section{II - EL VALOR DE LA COHERENCIA}

El tipo de argumento esgrimido con mayor frecuencia en favor del valor racional de la coherencia en la razón práctica, se funda en la

\footnotetext{
${ }^{5}$ E.g. MacCormick, N. (2005) Rhetoric and the Rule of Law (Oxford, OUP), 193. Para mis fines no es necesario hacer mucho con la aparente contradicción entre mi afirmación de que la intensionalidad se relaciona con aspectos intrínsecos de los elementos conectados y la afirmación de MacCormick acerca de la posibilidad de una conexión meramente instrumental entre un elemento y el valor que torna los elementos en un conjunto coherente. Mi uso de la palabra "intrínseco" en este contexto comprendería también conexiones instrumentales, desde que esa relación instrumental pueda ser considerada un atributo potencial del elemento.
} 
premisa de que la búsqueda de un significado unificado (incluso el significado de la vida humana) es un imperativo de la razón. Alexy y Peczenick llegan a afirmar que el propio concepto de pensamiento racional podría ser explicado como un intento de obtener un balance adecuado entre una serie de criterios de coherencia ${ }^{6}$. Por supuesto que esta forma de defensa del valor de la coherencia no es universalmente aceptada y, de hecho, fue frontalmente atacada por Joseph Raz en un famoso texto ${ }^{7}$.

Amaya propone una vía de justificación para el valor de la coherencia que busca evitar la necesidad de tomar una posición en este debate sobre una teoría general de la racionalidad. Ella ofrece tres argumentos para justificar el valor de la coherencia: un argumento epistémico general, uno de carácter práctico y uno fundado en lo que llama "razones constitutivas". Considero que el primer argumento no logra éxito en dicha tarea, pero inaugura una línea de argumentación promisoria. Veamos.

El argumento epistémico general busca partir de una conexión entre la coherencia de un conjunto de creencias y su verdad. Esta conexión no es una inferencia lógica, sino una relación de mayor probabilidad. Ahora, en relación al requisito de no-contradicción, es evidente que la probabilidad de que un conjunto incoherente de creencias sea verdadero es inexistente y que la probabilidad de que un conjunto coherente de creencias sea verdadero es mayor o igual a cero. En este sentido tal vez se pueda hablar de una mayor probabilidad. Todavía, como vimos, se puede obtener consistencia entre elementos incoherentes (un vez que es sólo una condición necesaria, pero mas no suficiente, para la coherencia). O sea, esa forma de aumento de la probabilidad se obtiene con mera consistencia.

¿Pero es posible concluir que la coherencia (y no la mera consistencia) aumenta la probabilidad de verdad de un conjunto de creencias? El modo como Amaya conduce su argumento sobre este punto es interesante. Ella acepta que no hay cualquier conexión

${ }^{6}$ Alexy, R. y Peczenick, A. (1990) The concept of coherence and its significance for discursive rationality Ratio Iuris Vol 3, n. 1, 130, 144.

${ }^{7}$ Raz, J. (1994) The Relevance of Coherence in J. Raz Ethics in the Public Domain (Oxford, Clarendon Press), 277, 300. 
necesaria entre coherencia y verdad y observa que, en relación a esa conexión necesaria, argumentos sobre coherencia se quedan en una situación que no es peor que la situación de cualquier otra relación entre un argumento y la verdad de sus conclusiones. Es posible concluir, por lo tanto, que los argumentos sobre la imposibilidad de una perfecta conductividad a la verdad no nos habilitan para llegar a la conclusión de que sea falsa la tesis de que hay mayor probabilidad de veracidad en conjuntos de creencias coherentes.

Desde este argumento, su conclusión es negativa: "no hay razones concluyentes para rechazar el valor de los estándares coherentistas de la justificación como herramientas para la búsqueda de la verdad" (Amaya, 51). Amaya lista algunos intentos de fundar una conexión entre coherencia y verdad, pero su conclusión no se construye sobre esas tentativas.

Todavía, la cuestión que debe ser respondida para que se pueda hablar de una justificación epistémica para el valor de la coherencia no queda respondida: ¿por qué un conjunto intensionalmente unificado de creencias es probablemente más verdadero que un número similar de creencias sin cualquier vínculo intensional (no-contradictorias, por supuesto)?

A mi juicio no se puede ofrecer una respuesta general para esta pregunta. Todavía es posible ofrecer argumentos epistémicos (o, por lo menos, con consecuencias epistémicas) a favor de la coherencia en contextos más específicos como, por ejemplo, el contexto de creencias jurídicas o morales. Las razones para esto se relacionan no simplemente con teorías epistémicas sobre la verdad moral, pero se fundan en cuestiones morales substantivas. Aunque no es posible en este comentario presentar una defensa completa de estas razones morales substantivas, es necesario por lo menos presentar la estructura general del argumento que pienso puede proveer una justificación como esa.

Para que se pueda evaluar de manera más precisa el tipo de argumento que pienso es el adecuado para justificar el valor de la coherencia en cuestiones de razón práctica, es importante notar que incluso quienes objetan a la coherencia en estos campos aceptan un cierto grado de unidad entre razones (o creencias, normas, valores, etc.) morales y jurídicas. De hecho, en este nivel de abstracción la tesis de 
Alexy y Peczenick mencionada más arriba (de que la coherencia está intrínsicamente conectada a la razón) parece (tal vez trivialmente) verdadera. Por ejemplo, en mi amplia definición de la segunda ventaja racional de la coherencia (unidad), la misma concepción de Raz sobre las razones de autoridad se podría considerar coherente. De hecho, Raz no objeta el hecho de que haya una conexión intensional entre las normas producidas por autoridades legítimas. La tesis de la justificación normal es precisamente una forma de dar unidad de sentido a un grupo de directivas. La objeción de Raz a la coherencia se dirige a una concepción particular de esta conexión intensional, a saber, aquella según la cual la conexión entre las normas jurídicas consistiría en la unidad de valor moral presente en estas normas. Para Raz, la conexión intensional se obtiene a partir del hecho que, cuando se trata de autoridades legítimas, hay una mayor probabilidad de hacer la cosa adecuada si el agente opta por seguir las directivas emitidas por la autoridad ${ }^{8}$. No hay ningún valor moral substantivo (o grupo de valores morales substantivos) que conecte todas las directivas emitidas por la autoridad. De hecho, de acuerdo con Raz, la autoridad puede emitir directivas completamente contrarias sin que la probabilidad de hacer la cosa adecuada respetando las directivas se torne menor que la probabilidad de hacer la cosa adecuada actuando conforme mis propias razones. El argumento de Raz, por lo tanto, no sería una objeción a la idea de que hay una ventaja racional en la unidad de elementos de un conjunto dado (de creencias, normas, etc.). Al contrario, su argumento parece suponer una unidad intensional entre razones para la acción que resultan de los actos de autoridad.

Sin embargo, creo que la mejor (efectivamente, la única) manera de justificar el valor de la coherencia en cuestiones de razón práctica resulta de una condición substantiva para la realización del agente moral $^{9}$. La realización moral del sujeto está condicionada a la

\footnotetext{
${ }^{8}$ Raz, J. (1994) 'Authority, law and morality' in J. Raz, Ethics in the Public Domain: Essays in the morality of law and politics (Oxford: Clarendon Press), 214.

${ }^{9}$ Mi objeción a la tentativa de Raz de fundar la autoridad de las razones jurídicas en su concepción de autoridad legítima se encuentra en el capítulo 4 de mi Being Apart from Reasons (Dordrecht, Springer, 2006) en cuanto que una versión un poco más desarrollada de los argumentos que sigue sobre coherencia narrativa se encuentra en Michelon (2010) 'Princípios e Coerência na 
unidad narrativa de su vida, o sea, a la posibilidad de que las acciones del sujeto se conduzcan a una concepción determinada del bien. Nada impide que esta concepción sea compleja, articulando un conjunto de bienes y valores que no se relacionan solamente como derivaciones lógicas unos de los otros. Estos bienes pueden incluso ser de tipos diferentes, unos instrumentales y otros intrínsecos, pero todos se deben relacionar con una concepción de realización humana ${ }^{10}$. Conforme a Charles Taylor,

(...), en la medida que vemos algún sentido en nuestras vidas, o en las vidas que estamos intentando llevar, estaremos relacionando los diferentes bienes que buscamos no sólo en relación a su diferente importancia, pero también en relación al modo como se adecuan, o no, como conjunto, en el desenvolvimiento de nuestras vidas ${ }^{11}$.

Esta búsqueda de sentido, que se realiza a través de una constante "interpretación creativa de nosotros mismos"12, es un reflejo psicológico de la importancia objetiva de concebir una vida de forma narrativamente coherente. Ese imperativo de buscar la coherencia narrativa relacionando acciones con ciertos bienes apunta a favor de la necesidad de una antropología filosófica (i.e. de una teoría del bien humano). Es interesente notar que ese tipo de teoría se adecua confortablemente a una concepción metafísica realista, algo que Amaya considera una teoría menos plausible (que el constructivismo) sobre la verdad de los enunciados normativos (Amaya,51). Si yo estoy en lo

\footnotetext{
Argumentação Jurídica' in Macedo, R. Direito e Interpretação: Racionalidades e Instituições (São Paulo: Saraiva).

${ }^{10}$ Véase la relación entre bienes de eficacia y bienes de excelencia en MacIntyre A. (1988) Whose Justice? Which Rationality? (London, Duckworth) p. 32 ss.

11 "But insofar as we have some sense of our lives, or what we are trying to lead, we will be relating the different goods we seek not just in regard to their differential importance, but also to the way they fit, or fail to fit, together in the unfolding of our lives." Taylor, C. (1997) Leading a Life in Ruth Chang (ed) Incommensurability, Incompatibility, and Practical Reason (Cambridge-MS, Harvard University Press), 182.

${ }^{12}$ McCabe, H. (1968), Law, Love, Language (London, Continuum), p. 143.
} 
correcto, al creer que la unidad narrativa de la vida humana se funda en una antropología filosófica, es posible que el realismo sea una mejor justificación para el valor de la coherencia, al menos en relación con cuestiones de razón práctica.

\section{III - LOS LÍMITES DE LA COHERENCIA}

En diversos pasajes de su artículo, Amaya demuestra (a mi juicio de modo convincente) la insuficiencia de la coherencia, pasando revista a diversas razones por las cuales la constatación de que haya coherencia entre diferentes creencias (o normas, o valores, etc.) no es suficiente para garantizar ni la verdad de estas creencias, ni la racionalidad de las mismas. Que la coherencia no garantice la verdad de una creencia no es particularmente sorprendente. Lo que es sorprendente es que la certeza haya, por un largo período, dejado de ser un predicado psicológico sobre el grado de seguridad que una persona tiene sobre sus creencias y se haya vuelto una condición para que se pueda afirmar la veracidad de estos enunciados. En efecto, desde Descartes distintos filósofos han sido acosados por las implicaciones epistemológicas de la posibilidad de error. ¿Puede haber conocimiento, sin que haya certeza (i.e. la imposibilidad de estar equivocado)? Sin embargo, yo creo que la cuestión sobre la garantía de veracidad no es la más filosóficamente interesante.

Mucho más interesante es la cuestión sobre la relación entre coherencia y racionalidad. En la primera sección de este comentario, presenté dos ventajas racionales de la coherencia: (a) decir que hay coherencia entre, por ejemplo, proposiciones, significa que no hay una contradicción entre ellas, y (b) coherencia implica una unidad intensional de los elementos coherentes.

Amaya ofrece razones por las cuales estas ventajas necesitan ser complementadas. En primer lugar, en la dinámica del uso de argumentos de coherencia, hay una fase crucial que no puede ser decidida a partir de los criterios de no-contradicción y de unidad. En la primera fase son generados o identificados los elementos relevantes; en la segunda, diversas alternativas que reconducen estos elementos a una 
unidad no-contradictoria son construidas; en la tercera, distintos argumentos son aducidos a favor de una u otra de las alternativas (Amaya, 29). La tercera fase es particularmente problemática, una vez que es perfectamente posible que los criterios de no-contradicción y de unidad sean insuficientes para decidir racionalmente cuál sea la mejor manera de conectar los elementos. En otras palabras, la coherencia no proporciona " $[. .$.$] ningún criterio de decisión para elegir entre distintos$ conjuntos alternativos de creencias que son igualmente coherentes" (Amaya, sección 8). El problema no se plantea habitualmente, pero está potencialmente presente en cualquier discusión sobre la coherencia de un grupo de elementos.

Según la interpretación de MacIntyre, el objetivo del libro primero de la República de Platón sería precisamente demostrar los límites de una argumentación cuyo único criterio de racionalidad fuera la no-contradicción (MacIntyre 1988:71-73). Creo que, si aceptamos la interpretación de MacIntyre, debemos concluir que también la unidad de los elementos sería insuficiente (o sea, que la coherencia como un todo es insuficiente en cuanto criterio de racionalidad. En este texto, la reacción de Trasímaco a la mayéutica socrática es simplemente la de abrir la mano de todas las proposiciones que estén en conflicto con su tesis principal sobre la justicia (de que la justicia es lo que aprovecha al más fuerte)). Sócrates, como siempre hace en los diálogos platónicos, pregunta la opinión de Trasímaco sobre una serie de temas que no están aparentemente conectados con la concepción de justicia de Trasímaco (i.e. intenta inventariar ciertas intuiciones morales de Trasímaco) y después procura demostrar cómo estas intuiciones morales contradicen su tesis central sobre la justicia como el provecho del más fuerte. Aún según MacIntyre, el (casi absoluto) silencio de Trasímaco después del libro primero es una forma literaria encontrada por Platón para expresar el hecho de que Sócrates no logró derrotar la concepción de justicia defendida por Trasímaco, de forma que el argumento posterior del diálogo nada dice respecto al último.

O sea, Trasímaco adopta una de las (perfectamente legítimas) estrategias identificadas por Amaya, la "contracción" (que en algunas ocasiones es precedida de un intento de reinterpretación). Sócrates, a su vez, adopta estrategias de adición, en su intento de incluir nuevos 
elementos en el conjunto de creencias que debe ser racionalmente justificado. Pero el límite de la adición es precisamente la contracción.

Amaya inteligentemente identifica un problema en este punto: ¿Cómo escoger racionalmente entre dos teorías rivales que son (o pueden ser elaboradas, mediante las estrategias de contracción y reinterpretación) perfectamente coherentes internamente?

En el pasado, Amaya ha defendido una salida ecléctica para este problema, esto es, una combinación de ciertos deberes epistémicos que deben ser cumplidos por el investigador con un conjunto de virtudes intelectuales que llevarían a este investigador a no manipular las estrategias con el objetivo de obtener los resultados que le gustaría obtener. En el artículo que aquí se comenta, Amaya parece más inclinada a confiar solamente (o por lo menos primariamente) en el hecho de que el investigador posea ciertas virtudes intelectuales.

El problema aquí no está bien planteado. Del problema de la insuficiencia de la coherencia surgen dos problemas distintos y yo creo que la solución propuesta por Amaya puede contribuir para la solución de uno de estos problemas. En primer lugar, se pone la cuestión de cómo evitar el abuso subjetivo (por el investigador) de las estrategias de coherencia ¿Hay que proponer un conjunto de reglas de conducta para el investigador? ¿Quizá sea necesario que el investigador desarrolle un cierto tipo de carácter, combinando una serie de virtudes? ¿O tal vez debamos considerar la posibilidad de una combinación de ambas estrategias? Pero, en segundo lugar, surge el (creo más fundamental) problema de los criterios de racionalidad que nos permitan juzgar cuál de las posibles explicaciones coherentes es racionalmente superior. La cuestión del control del abuso subjetivo de los mecanismos de coherencia y la cuestión de los criterios racionales para adjudicar el valor racional de dos explicaciones rivales de la coherencia de ciertos elementos, están relacionadas de tal modo que la respuesta a la segunda tiene implicaciones en relación a la primera. Por lo tanto, conviene empezar la discusión por la segunda.

Una respuesta simple a la cuestión de la insuficiencia racional de la coherencia consiste en ofrecer criterios adicionales de racionalidad. Yo creo que una serie de criterios adicionales de racionalidad (por lo menos de racionalidad práctica) puede ser encontrada en torno de la 
noción de no-circularidad (MacIntyre 1988:79-81). La no-circularidad impone a una teoría (y no simplemente a un teórico) requisitos adicionales para que pueda ser considerada racional. Por ejemplo, una teoría es más racional que las rivales, si incorpora en sí una explicación del error (o de los errores) de las teorías rivales. MacIntyre ha propuesto una serie de otros requisitos para que una teoría pueda ser considerada no-circular (e.g. la incorporación en la teoría de una explicación de su valor evolutivo, en dirección a una arché).

Este imperativo de no-circularidad formaliza la necesidad de enfrentamiento entre explicaciones rivales. Introduce, como vimos, así mismo una necesidad de hacer las teorías rivales parte del objeto a ser explicado por cada teoría. Pero este enfrentamiento no se limita a esto. De hecho, teorías de la racionalidad (como la que Aristóteles expone en la Topica) son precisamente intentos que buscan identificar las "rules of engagement" entre diversas teorías. La importancia de la no-circularidad no debe ser subestimada. Esta no es tan solo un requisito más de racionalidad entre muchos otros. En conjunto con la coherencia, la nocircularidad es uno de los principios estructurales de la razón práctica.

Ahora bien, es posible argumentar que este otro requisito de racionalidad no es el mejor criterio adicional de racionalidad. Es posible defender la tesis de que el criterio para juzgar cuál explicación rival (coherente) debería ser preferida corresponde al juzgamiento del investigador virtuoso. Esta fue, sin duda, la interpretación que fue dada a los pasajes de la Ética aristotélica donde se afirma que el hombre prudente (el Phronimos) es el criterio y medida de corrección en cuestiones de razón práctica ${ }^{13}$. No creo que esta noción del sujeto virtuoso como criterio de corrección sea adecuada para el tipo de problema que aquí estamos tratando, vale decir, el problema de los criterios de racionalidad que permiten escoger racionalmente entre explicaciones rivales de la unidad de ciertos elementos (creencias, normas, etc. $)^{14}$. Sin

${ }^{13}$ Aritóteles Ética a Nicómaco (1106b36). Para una discusión de los criterios personales véase Aubenque, P. (1963) La Prudence chez Aristote (Paris, Quadrige/PUF, 2nd ed. 1997), 33-41.

${ }^{14}$ Sin embargo, creo que la noción de sujeto-criterio puede ser relevante en situaciones en las cuales lo que se indaga es la coherencia de la correcta percepción de elementos. 
embargo, no creo que este sea el espíritu subyacente a la tesis defendida por Amaya sobre la importancia de una concepción de responsabilidad epistémica fundada en una ética de virtudes. Me parece que su tesis se relaciona al segundo problema identificado arriba, i.e. la del control del abuso subjetivo de los mecanismos de coherencia.

En relación a este segundo problema, ciertas virtudes epistémicas son, efectivamente, esenciales. Para tomar apenas algunas de esas virtudes, enumeradas por Roberts y Woods ${ }^{15}$, que uno posea un real amor por el conocimiento (un amor mayor del que tiene, por ejemplo, por su reputación científica o por la acumulación de capital) es un estado de cosas conducente a una utilización no abusiva de los mecanismos de adición, constricción y reinterpretación. Otras virtudes pueden ser añadidas al amor por el conocimiento: coraje intelectual, constancia, humildad, etc. Todas estas virtudes, tomadas en conjunto, disminuyen mucho la posibilidad de abuso (aún cuando sea imposible garantizar que ciertos abusos jamás acontecerán). En esto Amaya y yo estamos en pleno acuerdo. Todavía me parece que la posición ecléctica, originalmente defendida por Amaya, puede ser defendida de la objeción que ha hecho que ella cambiase su posición a favor de una salida centrada en las virtudes epistemológicas.

Amaya cree (acertadamente, a mi juicio) que hay un golfo muy largo entre éticas de la virtud y éticas deontológicas (Amaya, 36). De esta premisa, ella deriva la conclusión de que no es posible (o tal vez simplemente que no es necesaria) una conjugación entre deberes epistemológicos y virtudes epistemológicas. Sin embargo, este argumento parece presuponer que una ética de las virtudes no reservaría espacio conceptual a los deberes. Esta presuposición, a la vez, puede ser interpretada de un modo fuerte o débil. La versión débil afirma que las virtudes solo excluyen la utilidad de identificar deberes morales, pero no es posible reducir virtudes a complejas concatenaciones de deberes. En esta versión, hay espacio conceptual para los deberes y, por lo tanto, la contraposición entre éticas deontológicas y éticas de la virtud es suficiente para excluir la charla sobre deberes epistemológicos.

\footnotetext{
${ }^{15}$ Roberts, R. and Wood, W. J. (2007) Intellectual Virtues (Oxford, Clarendon Press).
} 
La versión fuerte es que no existe un espacio para los deberes en una ética de virtudes, pero esta tesis es evidentemente falsa en la medida que una ética de las virtudes es perfectamente compatible con la asignación de valor a la noción de deber. Algunos deberes pueden ser elementos necesarios en procesos pedagógicos de enseñanza de las virtudes, procesos que son, a su turno, necesarios para el desarrollo de las virtudes. Ciertos deberes pueden también tener un papel en una ética de las virtudes que admita la importancia de obedecer actos de autoridad como, por ejemplo, actos obligatorios según la ley. Con relación específicamente a cuestiones epistemológicas, la identificación de esta clase de deberes tiene un papel pedagógico central, identificando aquellos casos centrales cuya ausencia impediría desarrollar la virtud intelectual. Lo que se sugiere aquí no es una competencia entre dos concepciones éticas rivales, pero sí una cooperación entre dos instrumentos (deberes y virtudes) al interior de una versión de la ética de las virtudes. 